\title{
Comparison of prognosis between surgical resection and transarterial chemoembolization for patients with solitary huge hepatocellular carcinoma
}

\author{
Cheng-Yi Wei ${ }^{1}$, Po-Chun Chen ${ }^{1,2}$, Gar-Yang Chau ${ }^{2,3}$, Rheun-Chuan Lee ${ }^{2,4}$, Ping-Hsien Chen ${ }^{2,5,6}$, \\ Teh-Ia Huo ${ }^{1,7,8}$, Yi-Hsiang Huang ${ }^{1,9}$, Yu-Hui Su ${ }^{10}$, Ming-Chih Hou ${ }^{1,2}$, Jaw-Ching Wu ${ }^{8,9}$, Chien-Wei Su ${ }^{1,2}$ \\ ${ }^{1}$ Division of Gastroenterology and Hepatology, Department of Medicine, Taipei Veterans General Hospital, Taipei; ${ }^{2}$ Faculty of Medicine, School \\ of Medicine, National Yang-Ming University, Taipei; ${ }^{3}$ Division of General Surgery, Department of Surgery, ${ }^{4}$ Department of Radiology, ${ }^{5}$ Endoscopy \\ Center for Diagnosis and Treatment, Taipei Veterans General Hospital, Taipei; ${ }^{6}$ Institute of Biophotonics, ${ }^{7}$ Institute of Pharmacology, National \\ Yang-Ming University, Taipei; ${ }^{8}$ Department of Medical Research, Taipei Veterans General Hospital, Taipei; ${ }^{9}$ Institute of Clinical Medicine, National \\ Yang-Ming University, Taipei; ${ }^{10}$ Department of Accounting, School of Business, Soochow University, Taipei \\ Contributions: (I) Conception and design: CY Wei, PC Chen, GY Chau, RC Lee, YH Su, CW Su; (II) Administrative support: GY Chau, RC Lee, \\ PH Chen, TI Huo, YH Huang, MC Hou, JC Wu, CW Su; (III) Provision of study materials or patients: GY Chau, RC Lee, PH Chen, YH Huang, \\ MC Hou, CW Su; (IV) Collection and assembly of data: CY Wei, PC Chen, PH Chen, CW Su; (V) Data analysis and interpretation: CY Wei, PC \\ Chen, YH Su, CW Su; (VI) Manuscript writing: All authors; (VII) Final approval of manuscript: All authors. \\ Correspondence to: Chien-Wei Su, MD, PhD. Division of Gastroenterology and Hepatology, Department of Medicine, Taipei Veterans General \\ Hospital, No. 201, Sec. 2, Shipai Rd., Peitou District, Taipei 11217. Email: cwsu2@vghtpe.gov.tw.
}

Background: For patients with solitary huge (>10 $\mathrm{cm}$ in size) hepatocellular carcinoma (HCC) and without major vascular invasion, the treatment efficacy between surgical resection (SR) and transarterial chemoembolization (TACE) is not well studied. We aimed to compare the prognoses between SR and TACE for patients with solitary huge HCC.

Methods: We enrolled 143 patients with treatment-naïve, solitary HCC (>10 cm) who had received either SR or TACE treatment between 2007-2016. Factors of overall survival (OS) were analyzed by multivariate analysis. Propensity scores matching (PSM) method was adopted to adjust baseline demographic differences for further analysis.

Results: Ninety patients underwent SR and 53 patients received TACE. After a median follow-up of 17.0 (interquartile range 7.7-45.6) months, 83 patients had died. The cumulative 5 -year OS rate was $44.7 \%$ and $11.7 \%$ for the SR group and the TACE group, respectively $(\mathrm{P}<0.001)$. A multivariate analysis showed that TACE [hazard ratio (HR): 3.515, 95\% confidence interval (CI): 2.202-5.610, $\mathrm{P}<0.001$ ], and albuminbilirubin (ALBI) grade $>1$ (HR: 2.181, 95\% CI: 1.343-3.543, $\mathrm{P}=0.002$ ) were the independent risk factors associated with poorer OS. After PSM, 37 pairs of matched patients were selected from each treatment arm. After matching, patients who underwent SR still evinced a significantly higher OS than did those who underwent TACE $(\mathrm{P}=0.010)$.

Conclusions: SR provided a better OS than did TACE for patients with solitary huge $(\geq 10 \mathrm{~cm}) \mathrm{HCC}$. As such, SR is recommended as the therapeutic priority for these patients.

Keywords: Hepatocellular carcinoma (HCC); prognosis; propensity score matching; surgical resection; transarterial chemoembolization

Submitted Sep 26, 2019. Accepted for publication Dec 24, 2019.

doi: 10.21037/atm.2019.12.157

View this article at: http://dx.doi.org/10.21037/atm.2019.12.157 


\section{Introduction}

Hepatocellular carcinoma (HCC) is the second most lethal malignancy after pancreatic cancer (1), being responsible for 745,500 deaths worldwide in 2012 and ranking as the second and sixth most common cause of cancer among men and women, respectively (2). According to the current standards of HCC management, treatments are classified as either curative or non-curative (3). Curative treatments include surgical resection (SR), liver transplantation, and local ablation therapies, which could provide a median overall survival (OS) of over five years for HCC patients after treatment (1). Non-curative treatments include transarterial chemoembolization (TACE), transarterial radioembolization, external beam radiotherapy, and systemic therapy (e.g., molecular target therapy, immunebased therapy). For patients with huge $(>10 \mathrm{~cm}$ in diameter) HCC, local ablation is unfeasible because the tumor size exceeds physical limitation. Liver transplantation is also inapplicable given that the size exceeds the current criteria (4-6). Consequently, SR and TACE are more popular alternatives in this clinical context.

However, HCC patients with a larger tumor size displayed a higher incidence of vascular invasion and satellite nodules relative to those with a smaller tumor (7). Considering that vascular invasion is a major risk factor for tumor recurrence and poor OS after surgery (8-11), the indication and treatment efficacy of SR for patients with huge HCC is a topic of debate. Generally, Asian countries have adopted more liberal application of SR for HCC $(12,13)$, whereas the current guidelines for the management of HCC set by the European Association for the Study of the Liver (EASL) limited SR to patients with solitary HCC, well-preserved liver function, normal portal pressure and serum bilirubin levels, good performance status, and without extra-hepatic metastasis or vascular invasion (14). However, there is a growing body of evidence suggesting that SR could yield favorable outcomes for HCC patients with portal hypertension, size exceeding $5 \mathrm{~cm}$, multiple tumors, macroscopic vascular invasion, or even for those presenting with solitary extra-hepatic organ involvement (7,15-22). Another widely-applied classification system in Asia, Hong Kong Liver Cancer (HKLC), adopted more liberal criteria to SR (23).

Theoretically speaking, in the absence of distant metastasis and major vascular invasion, a clear-margin resection seems to serve as a curative therapy regardless of tumor size and number $(16,21)$. We hypothesized that, for patients with solitary huge HCC in addition to wellpreserved liver function, SR may still provide a survival advantage over the guideline-endorsed treatment TACE. To test this hypothesis, the long-term prognoses between SR and TACE for patients with single huge HCC were compared.

\section{Methods}

\section{Patients}

A prospectively conducted and retrospectively analyzed cohort study was conducted. The diagnosis of HCC was established according to the criteria set forth by the American Association for the Study of Liver Disease (AASLD) (24). All patients newly diagnosed with HCC at Taipei General Veterans Hospital were discussed to determine treatment strategy by a weekly-convened, multidiscipline HCC panel meeting attended by an oncologist, gastroenterologist, surgeon, radiologist, pathologist, oncoradiologist, and nursing personnel $(25,26)$. Following the meeting, treatment modality decision was shared with the patient and the physician after discussing the risks, benefits, complications, efficacies of the currently available treatments, and the multidisciplinary experts' recommendations. The demographic characteristics, treatment modalities, and prognoses of all patients with newly diagnosed HCC were prospectively recorded in the database for the multidisciplinary committee.

In this study, the inclusion criteria were as follows: (I) treatment-naïve HCC; (II) solitary tumor with size $\geq 10 \mathrm{~cm}$; (III) well-preserved liver function with Child-Pugh grade A or B; (IV) absence of major portal branch invasion by computed tomography scan or magnetic resonance imaging; (V) absence of distant metastases; and (VI) SR or TACE as the first treatment for HCC.

The study was conducted in accordance with the Declaration of Helsinki and current ethical guidelines. It was approved by the Institutional Review Board (IRB) of the Taipei Veterans General Hospital. Informed consent was obtained before the patient underwent SR or TACE. Patient information was anonymized prior to the initiation of this study.

\section{Statistical analysis}

The primary endpoint of this study was OS. All patients were followed up until either their final hospital visit, 


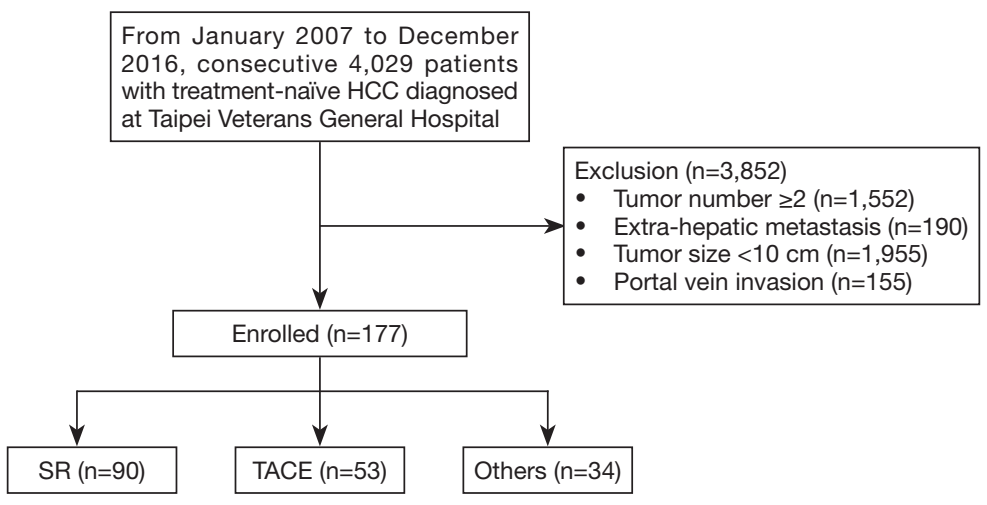

Figure 1 The study flow chart.

death, or December 31, 2017. Student's $t$-test and MannWhitney $U$ test were adopted for parametric and nonparametric distributed continuous variables, respectively. $\chi^{2}$ test and Fisher's exact test were applied for parametric and non-parametric nominal variables, respectively. KaplanMeier survival analysis was adopted for estimating OS after therapy. Cox proportional hazards model was employed to determine the factors associated with OS. Factors with a $\mathrm{P}$ value $<0.05$ in the univariate analysis were enrolled to multivariate analysis by a forward stepwise Cox's regression model.

We were aware that baseline demographic differences may confound OS analysis results. Therefore, a propensitymatched scoring (PSM) method was used to adjust for differences of demographic characteristics and tumor factors between the two groups of patients as previously described $(27,28)$. Subsequently, a one-to-one match between the SR and TACE groups was obtained using the nearestneighbor matching method. Survival analysis was repeated to compare the OS between SR and TACE amended from these confounding factors. A two-tailed $\mathrm{P}$ value $<0.05$ was defined as statistically significant. All statistical analyses were performed by IBM SPSS Statistics for Windows, Version 21.0 (IBM Corp., Armonk, NY, USA).

\section{Results}

\section{Baseline demographic characteristics}

From January 2007 to December 2016, a total of 4,029 patients were diagnosed with $\mathrm{HCC}$ in our center. Among these, 177 presented with a solitary huge HCC with a characteristic tumor size $\geq 10 \mathrm{~cm}$, and without main portal branch invasion or distant metastases. Ninety of these patients underwent SR, 53 patients underwent TACE, and the remaining 34 patients received another treatment such as radiotherapy, chemotherapy, or best supportive treatments (Figure 1).

As shown in Table 1, patients who underwent SR were younger than their TACE counterparts $(\mathrm{P}=0.001)$. Regarding the viral etiology, although results were not statistically significant, patients who had undergone SR displayed a trend of higher rate of hepatitis $\mathrm{B}$ virus (HBV) infection and lower rate of antibodies against hepatitis $\mathrm{C}$ virus $(\mathrm{HCV})$ in sera relative to those treated with TACE. Otherwise, gender, tumor size, and liver functional reserve did not differ significantly between the two groups.

\section{Factors related to $O S$}

There were two mortality cases within 30 days after TACE and no cases in the SR group. The 90 days mortality were six and two cases in the TACE group and in the SR group respectively. Following a median follow-up period of 17.0 (interquartile range IQR 7.7-45.6) months, 83 patients had died. As shown in Figure 2A, the 1-, 2-, 3-, and 5-year cumulative OS rate for patients who underwent SR and TACE were $78.1 \%$ vs. $45.1 \%, 67.5 \%$ vs. $27.6 \%$, $58.3 \%$ vs. $20.7 \%$, and $44.7 \%$ vs. $11.7 \%$, respectively. The median OS was 55.7 (95\% confidence interval CI: 23.4-88.1) months and 11.6 (95\% CI: 8.5-14.6) months for patients receiving SR and TACE, respectively. Patients treated with SR displayed better survival relative to those treated with TACE $(\mathrm{P}<0.001)$.

A univariate analysis disclosed that TACE, low platelet count, prothrombin time (PT) international normalized ratio $($ INR) $\geq 1.15$, serum bilirubin $\geq 1.0 \mathrm{mg} / \mathrm{dL}$, and albumin-bilirubin (ALBI) grade 2 or 3 (Figure $2 B$ ) were 
Table 1 Baselines demographics of enrolled patients

\begin{tabular}{|c|c|c|c|c|}
\hline & All patients $(n=143)$ & $\operatorname{SR}(n=90)$ & TACE $(n=53)$ & $\mathrm{P}$ \\
\hline Gender (M/F) (\%) & 117/26 (81.8/18.2) & 75/15 (83.3/16.7) & $42 / 11(79.2 / 20.8)$ & 0.540 \\
\hline BMI $\left(\mathrm{kg} / \mathrm{m}^{2}\right)$ & $23.6,21.5-25.9$ & $24.0,21.9-26.2$ & $23.0,20.9-25.4$ & 0.283 \\
\hline AFP (ng/mL) & $92.0,5.2-6,595.3$ & $87.7,4.3-6,944.2$ & $123.7,9.0-6,309.8$ & 0.583 \\
\hline HBsAg (+/-) (\%) & $82 / 54(60.3 / 39.7)$ & $57 / 30(65.5 / 34.5)$ & 25/24 (51/49) & 0.097 \\
\hline Anti-HCV (+/-) (\%) & $15 / 110(12 / 88)$ & $6 / 72(7.7 / 92.3)$ & 9/38 (19.1/80.9) & 0.056 \\
\hline MELD & $8.3,7.2-9.7$ & $8.0,7.1-9.7$ & $8.7,7.3-10.1$ & 0.746 \\
\hline ALBI & $-2.46,-2.79-2.11$ & $-2.49,-2.87-2.11$ & $-2.31,-2.67-1.96$ & 0.053 \\
\hline Albumin(mg/dL) & $3.7,3.4-4.1$ & $3.7,3.4-4.2$ & $3.6,3.3-4.0$ & 0.215 \\
\hline Bilirubin (U/L) & $0.80,0.58-1.15$ & $0.74,0.57-1.06$ & $0.92,0.63-1.51$ & 0.080 \\
\hline Platelet $\left(/ \mathrm{mm}^{3}\right)$ & $217,000,160,000-284,000$ & $226,000,167,000-297,000$ & $196,000,148,000-256,000$ & 0.129 \\
\hline PT INR & $1.07,1.02-1.14$ & $1.07,1.01-1.14$ & $1.07,1.02-1.18$ & 0.340 \\
\hline $\mathrm{Hgb}(\mathrm{mg} / \mathrm{dL})$ & $12.5,10.9-14.1$ & $12.8,11.4-14.5$ & $11.9,10.0-13.6$ & 0.167 \\
\hline BUN (mg/dL) & $16,12-19$ & $15,11-19$ & $17,12-20$ & 0.062 \\
\hline Creatinine (mg/dL) & $0.91,0.75-1.12$ & $0.90,0.74-1.10$ & $0.95,0.76-1.17$ & 0.594 \\
\hline ALT (U/L) & $43,28-71$ & $39,26-72$ & $46,32-71$ & 0.795 \\
\hline
\end{tabular}

Continuous variables are expressed as the median with 25th and 75th percentiles. SR, surgical resection; TACE, transarterial chemoembolization; BMI, body mass index; AFP, $\alpha$-fetoprotein; HBsAg, hepatitis B surface antigen; HCV, hepatitis C virus; MELD, model for end-stage liver disease; ALBI, albumin-bilirubin; PT INR, prothrombin time/international normalized ratio; HgB, hemoglobulin; ALT, alanine aminotransferase; GGT, $\gamma$-glutamyl transferase; ALKP, alkaline phosphatase.

associated with poorer OS for patients with a solitary huge HCC (Table 2). Multivariate analysis showed that TACE (HR: 3.515, 95\% CI: 2.202-5.610, $\mathrm{P}<0.001)$ and ALBI grade 2 or 3 (HR: 2.181 , 95\% CI: $1.343-3.543, \mathrm{P}=0.002$ ) were the independent factors predicting poorer OS. Subgroup analysis further revealed that, in the majority of patient subgroups, patients who underwent SR had a better OS than those who had received TACE, with the exception of female patients and patients with a PT INR $\geq 1.15$ (Figure 2C).

\section{Comparison of OS between SR and TACE after PSM analysis}

Because the baseline demographic characteristics were dissimilar between patients who underwent SR of TACE, a PSM analysis was performed to adjust for the differences between both of these groups. Subsequently, 74 patients (37 per group) were selected. As shown in Table 3, following the PSM, baseline demographics between both groups were well-matched, and survival analysis still indicated that SR 


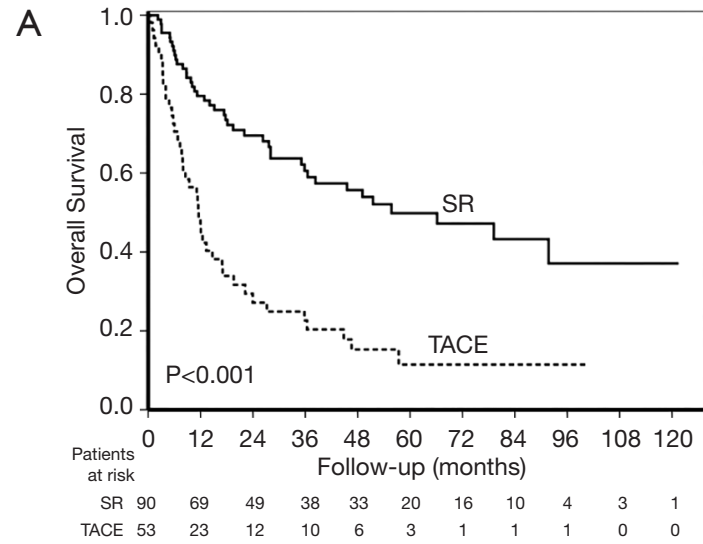

C

B

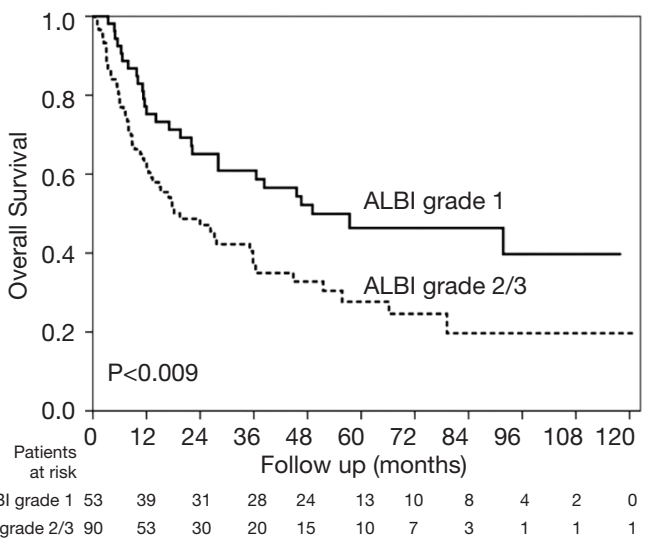

Age $\geq 65$
Age $<65$
Female
Male
BMI $\geq 24$
BMI $<24$
AFP $\geq 125$

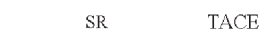

No. DEATH No. DEATH

$\mathrm{AFP}<125$

Size $\geq 12.5$

Size $<12.5$

$\begin{array}{llll}36 & 14 & 36 & 30\end{array}$

$\operatorname{HR}(95 \% \mathrm{CI})$
$3.962(2.067-7.5$

$\begin{array}{llll}54 & 27 & 17 & 5\end{array}$

$2.065(1.043-4.088)$

$2.548(0.778-7.764)$

$2.999(1.863-4.829)$

$2.294(1.081-4.871)$

$2.442(1.267-4.707)$

$2.773(1.519-5.063)$

$2.721(1.406-5.266)$

$2.092(1.122-3.902)$

$4.102(2.195-7.663)$

HBs negative

$3.891(1.740-8.703)$

HBs positive

Anti-HCV (+)

Anti-HCV (-)

ALBT $2 \& 3$

ALBI 1

Albumin $=3.5$

Albumin $\measuredangle 3.5$

Platelet $\leq 200 \mathrm{k}$

Platelet $>200 \mathrm{k}$

$\Pi \mathrm{NR} \geq 1.15$

$\mathrm{INR}<1.15$

Bilirubin $<1.0$

Bilirubin $\geq 1.0$

$\mathrm{Hb} \leq 12$

$\mathrm{Hb}>12$

Creatinine $\geq 1.0$

Creatinine $<1.0$

$A L T \geq 40$

ALT $<40$

$\mathrm{ALKP} \geq 100$

ALKP $<100$

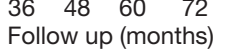

Patients 0

\begin{tabular}{|l}
$\mathrm{P}<0.010$ \\
$0 \quad 12$
\end{tabular}

$\begin{array}{rcccccccccc}\text { SR } & 37 & 29 & 19 & 14 & 12 & 8 & 5 & 3 & 0 & 0 \\ \text { TACE } & 37 & 16 & 10 & 9 & 5 & 3 & 1 & 1 & 1 & 0\end{array}$

$2.676(1.514-4.730)$

$5.588(1.804-28.800)$

$2.615(1.584-4.318)$

$3.357(1.933-5.830)$

$2.191(1.022-4.696)$

$2.838(1.619-4.975)$

$2.757(1.356-5.606)$

$3.912(2.031-7.537)$

$1.986(1.058-3.728)$

$1.888(0.842-4.233)$

$3.204(1.909-5.376)$

$2.922(1.700-5.111)$

$2.738(1.346-5.572)$

$2.431(1.260-4.692)$

$3.390(1.870-6.140)$

$4.277(2.084-8.778)$

$2.415(1.354-4.308)$

$2.980(1.272-5.271)$

$2.589(1.272-5.271)$

$2.504(1.380-4.544)$

5.544 (2.196-14.000)
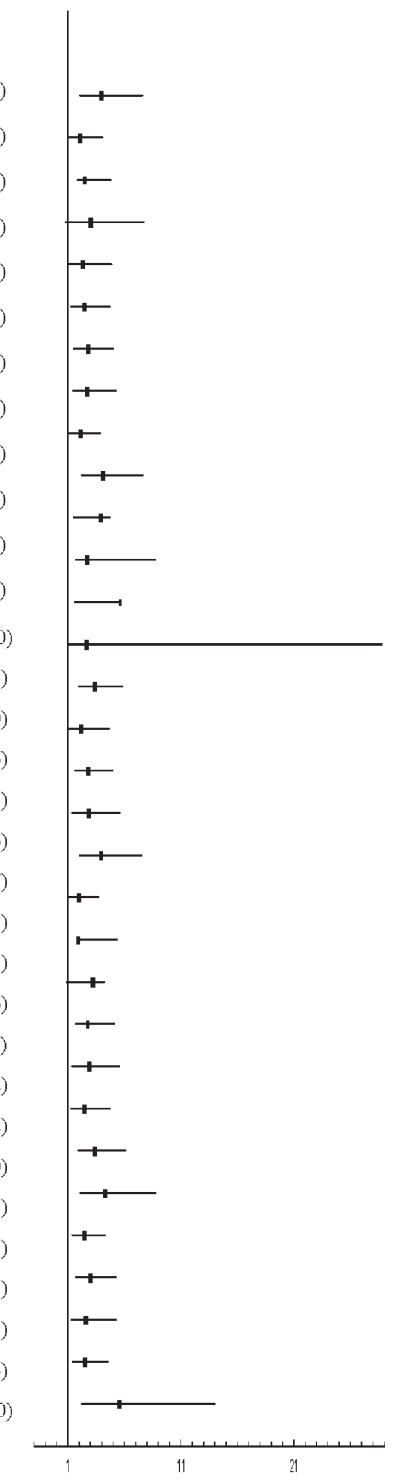

Figure 2 Comparison of OS rates between different treatment modalities, ALBI grades, and demographic characteristics and tumor factors. (A) Comparison of OS rates between patients having undergone SR and TACE before PSM. (B) Comparison of OS rates between patients with ALBI grade 1 and those with ALBI grade 2 or 3. (C) Subgroup analysis for the comparison of OS rates between patients having undergone SR or TACE. (D) Comparison of OS rates between patients having undergone SR or TACE after PSM. OS, overall survival; ALBI, albumin-bilirubin; SR, surgical resection; TACE, transarterial chemoembolization; PSM, propensity scores matching. 
Table 2 Factors associated with poor overall survival in HCC

\begin{tabular}{|c|c|c|c|c|c|}
\hline Variable & $\mathrm{N}(\%)$ & \multicolumn{2}{|c|}{ Univariate analysis } & \multicolumn{2}{|c|}{ Multivariate analysis } \\
\hline TACE/SR & $53 / 90(37.1 / 62.9)$ & $3.022(1.952-4.680)$ & $<0.001$ & $3.515(2.202-5.610)$ & $<0.001$ \\
\hline Age $(y / o)>65 / \leq 65$ & $68 / 75(47.6 / 52.4)$ & $1.310(0.851-2.018)$ & 0.220 & & \\
\hline Gender F/M & 26/117 (18.2/81.8) & $0.731(0.396-1.350)$ & 0.317 & & \\
\hline $\operatorname{AFP}(\mathrm{ng} / \mathrm{mL}) \geq 125 /<125$ & 68/72 (48.6/51.4) & $1.536(0.988-2.389)$ & 0.056 & & \\
\hline Size $(\mathrm{cm})>12.5 / \leq 12.5$ & 67/76 (46.9/53.1) & $0.817(0.530-1.259)$ & 0.360 & & \\
\hline $\mathrm{HBsAg}, \mathrm{N} / \mathrm{Y}$ & $54 / 82(39.7 / 60.3)$ & $0.644(0.404-1.026)$ & 0.064 & & \\
\hline Anti-HCV, N/Y & $110 / 15(87.7 / 12.3)$ & $0.804(0.399-1.619)$ & 0.541 & & \\
\hline Albumin $(\mathrm{mg} / \mathrm{dL}) \leq 3.5 />3.5$ & 88/53 (37.6/62.3) & $1.438(0.921-2.247)$ & 0.110 & & \\
\hline $\begin{array}{l}\text { Platelet }\left(/ \mathrm{mm}^{3}\right) \\
>200,000 / \leq 200,000\end{array}$ & 82/60 (57.7/42.3) & $1.603(1.042-2.466)$ & 0.032 & & \\
\hline PT INR $\geq 1.15 /<1.15$ & $34 / 108(23.9 / 76.1)$ & $1.904(1.181-3.069)$ & 0.008 & & \\
\hline Bilirubin $(\mathrm{mg} / \mathrm{dL}) \geq 1.0 /<1.0$ & 49/92 (34.8/65.2) & $1.652(1.061-2.573)$ & 0.026 & & \\
\hline $\mathrm{Hgb}(\mathrm{mg} / \mathrm{dL}) \leq 12 />12$ & $62 / 80(43.7 / 56.3)$ & $1.064(0.690-1.642)$ & 0.778 & & \\
\hline BUN $(\mathrm{mg} / \mathrm{dL}) \geq 20 /<20$ & 33/106 (23.7/76.3) & $0.951(0.568-1.592)$ & 0.848 & & \\
\hline Creatinine $(\mathrm{mg} / \mathrm{dL}) \geq 1.0 /<1.0$ & $53 / 89(37.3 / 62.7)$ & $1.339(0.864-2.075)$ & 0.192 & & \\
\hline
\end{tabular}

$\mathrm{Cl}$, confidence interval; SR, surgical resection; TACE, transarterial chemoembolization; BMI, body mass index; AFP, $\alpha$-fetoprotein; HBsAg, hepatitis B surface antigen; HCV, hepatitis C virus; MELD, model for end-stage liver disease; ALBI, albumin-bilirubin; PT INR, prothrombin time/international normalized ratio; $\mathrm{HgB}$, hemoglobulin; ALT, alanine aminotransferase; ALKP, alkaline phosphatase.

resulted in better OS than did TACE $(\mathrm{P}=0.010$; Figure $2 D)$.

\section{Comparison of OS between SR and TACE stratified by ALBI grade}

As treatment modality and ALBI grade were the independent factors correlated with poorer OS according to multivariate analysis, we further compared the prognoses between patients receiving SR or TACE by stratification by ALBI grade. In our cohort, although most patients were in Child-Pugh class A liver functional reserve (96.1\%), the proportion of ALBI grade was $37.3 \%, 57.7 \%$, and $4.9 \%$ for grade 1, 2, and 3, respectively. The distribution of ALBI grades in SR and TACE groups did not present statistically significant differences $(\mathrm{P}=0.386)$. Survival analysis showed that SR had better survival than TACE, both in terms of ALBI grade 1 (Figure $3 A, \mathrm{P}=0.030$ ) and ALBI grade 2 or 3 (Figure $3 B, \mathrm{P}<0.001$ ). For patients with ALBI grade 1 , the $1-, 2-, 3-$, and 5-year cumulative OS rate for patients having undergone SR vs. TACE were $83.2 \%$ vs. $58.8 \%, 77.3 \%$ vs. $39.2 \%, 71.1 \%$ vs. $39.2 \%$, and $58.0 \%$ vs. $24.5 \%$, respectively. For those with ALBI grade 2 or 3, the 1-, 2-, 3-, and 5-year cumulative OS rate for patients having undergone SR vs. TACE were $77.1 \%$ vs. $38.1 \%, 64.1 \%$ vs. $21.8 \%, 52.2 \%$ vs. $14.5 \%$, and $43.8 \%$ vs. $5.4 \%$, respectively.

\section{Factors of OS and recurrence-free survival in SR group}

Among the 90 patients who underwent SR, 75 patients $(83.3 \%)$ reached R0 resection (no cancer cells were found 
Table 3 Demographic data of HCC patients after propensity score matching

\begin{tabular}{|c|c|c|c|c|}
\hline Variable & Overall $(n=74)$ & SR $(n=37)$ & TACE $(n=37)$ & $\mathrm{P}$ \\
\hline Gender (M/F) (\%) & $58 / 16(78.4 / 21.6)$ & 29/8 (78.4/21.6) & 29/8 (78.41/21.6) & 1.000 \\
\hline BMI & $22.7,20.7-25.4$ & $22.9,20.9-26.0$ & $22.6,20.3-24.9$ & 0.624 \\
\hline AFP (ng/mL) & $57.8,4.6-1907.7$ & $17.9,3.9-1907.7$ & $97.9,5.8-4485.6$ & 0.511 \\
\hline HBsAg (+/-) (\%) & 39/34 (53.4/46.6) & $18 / 18(50 / 50)$ & 21/16 (56.8/43.2) & 0.731 \\
\hline Anti-HCV (+/-) (\%) & 9/65 (12.2/87.8) & $5 / 32(13.5 / 86.5)$ & 4/33 (10.8/89.2) & 0.469 \\
\hline MELD & $8.5,7.3-10.0$ & $8.0,7.1-10.1$ & $8.7,7.3-10.2$ & 0.712 \\
\hline ALBI & $-2.39,-2.68$ to -1.89 & $-2.40,-2.63$ to -1.97 & $-2.38,-2.69$ to 1.71 & 0.493 \\
\hline Platelet $\left(1,000 \mu \mathrm{L}^{-1}\right)$ & $213,160.7-270$ & $223,159.5-277$ & $209,162.5-262$ & 0.935 \\
\hline PT INR & $1.07,1.02-1.16$ & $1.07,1.02-1.14$ & $1.10,1.06-1.22$ & 0.473 \\
\hline Bilirubin (U/L) & $0.91,0.60-1.52$ & $0.80,0.58-1.36$ & $0.95,0.64-1.60$ & 0.328 \\
\hline $\mathrm{HgB}(\mathrm{mg} / \mathrm{dL})$ & $12.0,10.4-14.0$ & $11.6,10.9-14.3$ & $12.3,10.3-13.8$ & 0.681 \\
\hline BUN (mg/dL) & $16,12-19$ & $15,11.5-19.5$ & $16,11.8-19.0$ & 0.329 \\
\hline Creatinine (mg/dL) & $0.86,0.69-1.05$ & $0.88,0.69-1.02$ & $0.85,0.70-1.08$ & 0.858 \\
\hline ALT (U/L) & $45.5,30-81$ & $45,27.5-88$ & $47,32-76$ & 0.914 \\
\hline GGT (U/L) & $104,61-151.5$ & $91,61-122.5$ & $119.5,58.8-216$ & 0.634 \\
\hline
\end{tabular}

Continuous variables are expressed as the median with 25th and 75th percentiles. SR, surgical resection; TACE, transarterial chemoembolization; BMI, body mass index; AFP, $\alpha$-fetoprotein; HBsAg, hepatitis B surface antigen; HCV, hepatitis C virus; MELD, model for end-stage liver disease; ALBI, albumin-bilirubin; PT INR, prothrombin time/international normalized ratio; HgB, hemoglobulin; ALT, alanine aminotransferase; GGT, $\gamma$-glutamyl transferase.
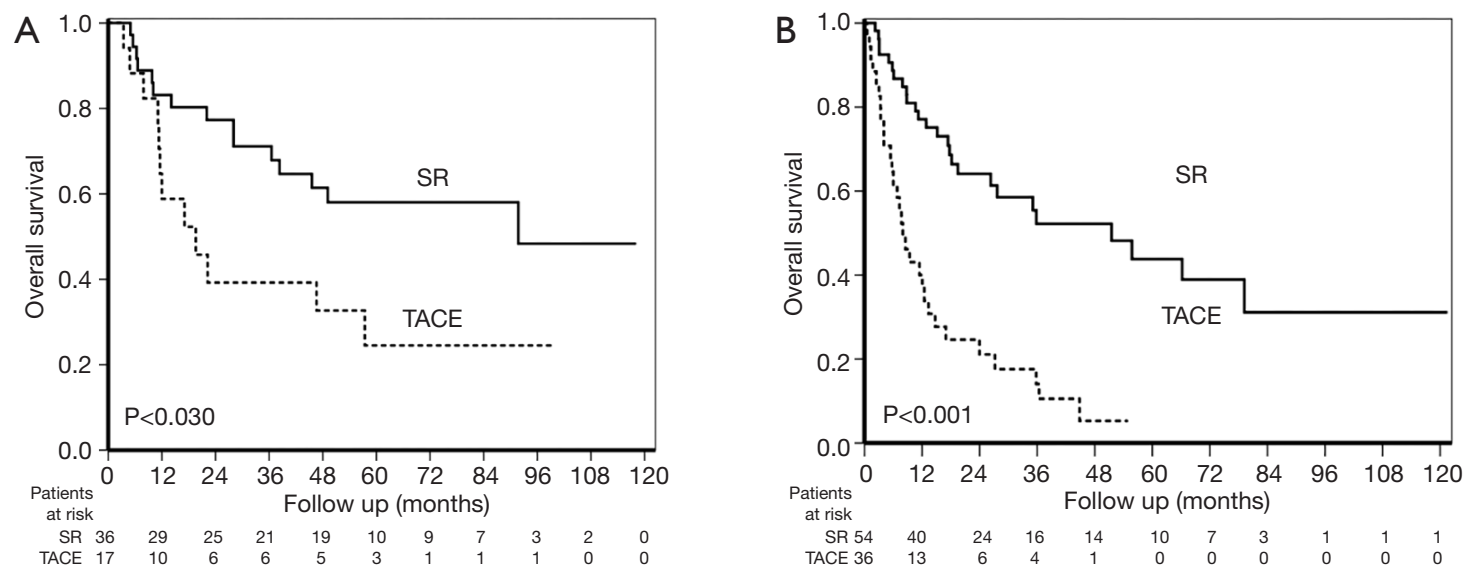

Figure 3 Comparison of OS rates between SR and TACE stratified by the ALBI grades. (A) ALBI grade 1; (B) ALBI grade 2 or 3 . OS, overall survival; SR, surgical resection; TACE, transarterial chemoembolization; ALBI, albumin-bilirubin. 

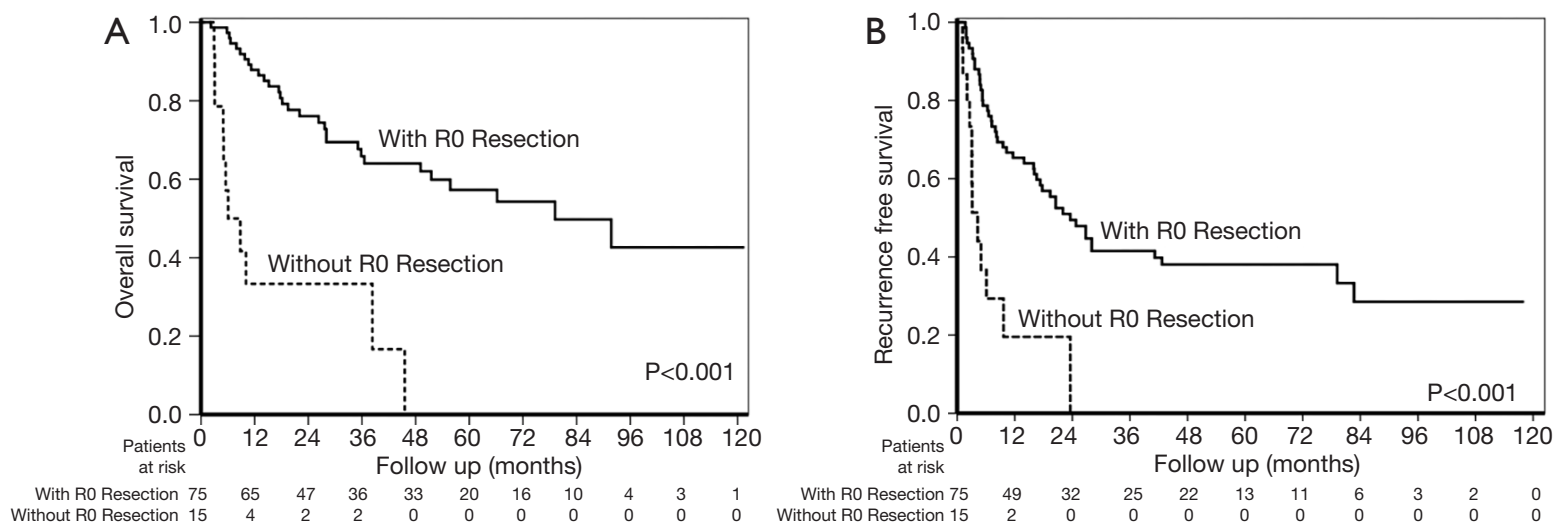

Figure 4 Comparison of prognoses between patients with and without R0 resection in the SR group. (A) OS rates; (B) RFS rates. SR, surgical resection.

in surgical margin, and tumor was completely resected). Pathological examination showed that $9(10 \%)$ patients had macrovascular invasion, and 79 patients $(87.8 \%)$ had microvascular invasion in their surgical specimen. When stratified by the status of R0 resection, the cumulative 1-, 2-, 3 -, and 5-year cumulative OS rates were $87.9 \%$ vs. $33.3 \%$, $76.1 \%$ vs. $33.3 \%, 65.9 \%$ vs. $33.3 \%$ and $57.3 \%$ vs. $0 \%$ in the R0 resection group and in the non-R0 resection group, respectively (Figure $4 A, \mathrm{P}<0.001$ ).

As shown in Table 4, a multivariate analysis disclosed the independent prognostic factors to poorer OS included ALBI grade 2 or 3 (HR: 4.252, 95\% CI: 1.998-9.049, $\mathrm{P}<0.001$ ) and no R0 resection (HR: 6.341, 95\% CI: $2.903-13.851$, $\mathrm{P}<0.001$ ).

Besides, 46 patients developed tumor recurrence after tumor resection. The median recurrence time was 5.7 (IQR 3.2-14.4) months. The median recurrence free survival (RFS) was 19.4 (IQR 13.1-25.7) months. The cumulative 1 -, 2-, 3-, and 5-year cumulative RFS rates were $65.3 \%$ vs. $19.6 \%, 49.4 \%$ vs. $0 \%, 41.5 \%$ vs. $0 \%$ and $38.1 \%$ vs. $0 \%$, respectively in the $\mathrm{R} 0$ resection group and in the non-R0 resection group, respectively (Figure $4 B, \mathrm{P}<0.001$ ).

A multivariate analysis showed that age $>65$ years (HR: 2.004, 95\% CI: 1.106-3.636, $\mathrm{P}=0.022$ ), serum alphafetoprotein (AFP) level $\geq 125 \mathrm{ng} / \mathrm{mL}$ (HR: 1.772, 95\% CI: 1.024-3.067, $\mathrm{P}=0.041$ ), ALBI grade 2 or 3 (HR: 5.055 , $95 \%$ CI: 2.687-9.511, $\mathrm{P}<0.001)$ and no R0 resection (HR: 5.458, 95\% CI: 2.644-11.264, $\mathrm{P}<0.001)$ were the independent factors predicting poorer RFS (Table 5).

\section{Discussion}

Our study reports several major findings. First, for patients with a solitary huge HCC, SR yielded a better long-term OS than did TACE, which was further substantiated by multivariate analysis, PSM analysis, and subgroup analysis. SR was found to be safe and may be served as the frontline treatment modality for such patients given that they are not contraindicated for the operation. Second, ALBI grade possessed a discriminatory capability to predict prognosis for patients with solitary huge HCC. It suggested that liver functional reserve still played a crucial role in determining the outcomes of patients with huge HCC, and it could provide an important reference to predict prognosis in this clinical setting.

Regarding evaluation of SR's suitability for patients with HCC, Eastern and Western medicinal perspectives might differ. For the Eastern world, large size, microscopic portal vein invasion, and presence of clinically significant portal hypertension (CSPH) do not alter surgeons' judgement on carrying out resection once a clear-cut margin is attainable and liver function is well-reserved $(12,16,29,30)$. Conversely, SR is discouraged in such clinical contexts by their Western counterparts (6). The present study showed that, for patients with solitary huge HCC in which the tumor size exceeded the Milan criteria, the median OS and 5-year cumulative OS rate in those having undergone SR were 55.7 months and $44.7 \%$, respectively. The results appeared satisfactory when compared to the previous results (31-33), 
Table 4 The univariate and multivariate with poor overall survival of patients in the SR group

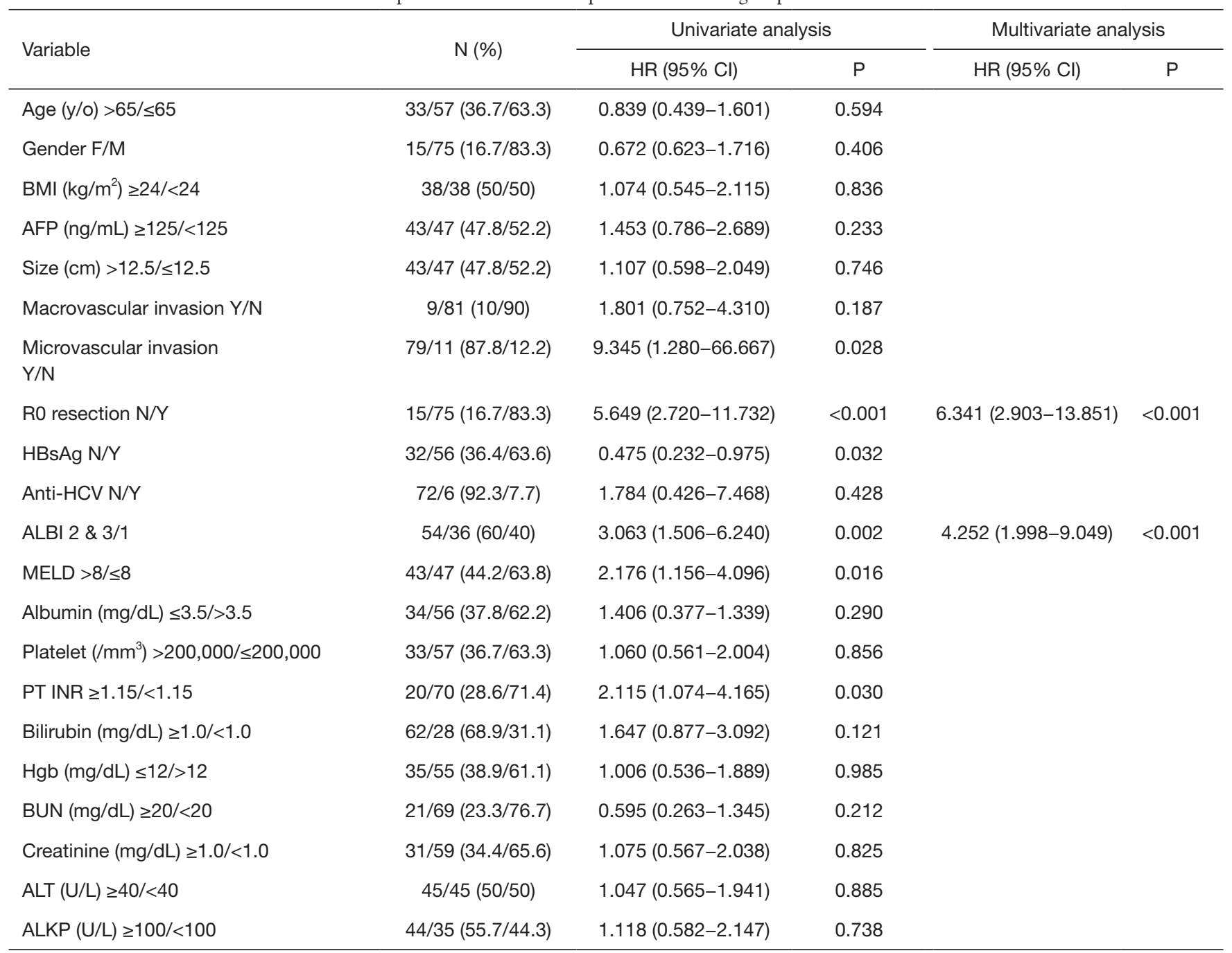

$\mathrm{Cl}$, confidence interval; SR, surgical resection; BMI, body mass index; AFP, $\alpha$-fetoprotein; HBsAg, hepatitis B surface antigen; HCV, hepatitis $C$ virus; MELD, model for end-stage liver disease; ALBI, albumin-bilirubin; PT INR, prothrombin time/international normalized ratio; $\mathrm{HgB}$, hemoglobulin; $\mathrm{ALT}$, alanine aminotransferase; ALKP, alkaline phosphatase.

and they were significantly better than those for patients who underwent TACE. Although major vascular invasion was excluded from our study, microscopic vascular invasion was expected in the majority of enrolled patients due to the tumor size surpassing $10 \mathrm{~cm}$ in diameter (7). Indeed, by pathological examination, macroscopic and microscopic vascular invasion were found in $10.0 \%$ and $87.8 \%$ of the patients in the SR group, respectively. However, both macroscopic and microscopic vascular invasion were not associated with poorer OS and RFS by multivariate analysis. By stead, ALBI grade and R0 resection could determine the outcomes of patients with solitary huge HCC after
SR. It implied that microscopic vascular invasion did not appear to hinder SR effectiveness relative to TACE if liver function was well preserved and $\mathrm{R} 0$ resection could be achieved. However, more prospective studies are warranted to elucidate the impact of microscopic vascular invasion on the outcomes of patients with solitary huge HCC and on the selection of the treatment modality for these patients.

The HKLC system classifies patients with HCC size exceeding $5 \mathrm{~cm}$, numbers less than 3 , and good liver functional reserve (Child-Pugh class A) as IIb (23). In their cohort, SR and TACE comprised the most common treatment modalities in this tumor stage. Moreover, SR 
Table 5 The univariate and multivariate with poor recurrence-free survival of patients in the SR group

\begin{tabular}{|c|c|c|c|c|c|}
\hline Variable & $\mathrm{N}(\%)$ & \multicolumn{2}{|c|}{ Univariate analysis } & \multicolumn{2}{|c|}{ Multivariate analysis } \\
\hline Age $(y / o)>65 / \leq 65$ & 33/57 (36.7/63.3) & $1.686(0.964-2.949)$ & 0.067 & $2.004(1.106-3.636)$ & 0.022 \\
\hline Gender F/M & 15/75 (16.7/83.3) & $0.463(0.199-1.078)$ & 0.074 & & \\
\hline BMI $\left(\mathrm{kg} / \mathrm{m}^{2}\right) \geq 24 /<24$ & $38 / 38(50 / 50)$ & $1.108(0.632-1.940)$ & 0.721 & & \\
\hline Size $(\mathrm{cm})>12.5 / \leq 12.5$ & $43 / 47(47.8 / 52.2)$ & $1.208(0.721-2.025)$ & 0.472 & & \\
\hline Macrovascular invasion $\mathrm{Y} / \mathrm{N}$ & $9 / 81(10 / 90)$ & $1.789(0.808-3.953)$ & 0.151 & & \\
\hline $\begin{array}{l}\text { Microvascular invasion } \\
\mathrm{Y} / \mathrm{N}\end{array}$ & 79/11 (87.8/12.2) & $3.344(1.199-9.346)$ & 0.021 & & \\
\hline ALBI $2 \& 3 / 1$ & $54 / 36(60 / 40)$ & 3.645 (1.992-6.673) & $<0.001$ & $5.055(2.687-9.511)$ & $<0.001$ \\
\hline MELD $>8 / \leq 8$ & 43/47 (44.2/63.8) & $1.385(0.826-2.322)$ & 0.217 & & \\
\hline Albumin $(\mathrm{mg} / \mathrm{dL}) \leq 3.5 />3.5$ & $34 / 56(37.8 / 62.2)$ & $1.712(1.011-2.907)$ & 0.045 & & \\
\hline $\begin{array}{l}\text { Platelet }\left(/ \mathrm{mm}^{3}\right) \\
>200,000 / \leq 200,000\end{array}$ & 33/57 (36.7/63.3) & $1.122(0.651-1.933)$ & 0.679 & & \\
\hline PT INR $\geq 1.15 /<1.15$ & 20/70 (28.6/71.4) & $1.477(0.795-2.746)$ & 0.217 & & \\
\hline Bilirubin $(\mathrm{mg} / \mathrm{dL}) \geq 1.0 /<1.0$ & $62 / 28(68.9 / 31.1)$ & $1.471(0.858-2.523)$ & 0.161 & & \\
\hline
\end{tabular}

SR, surgical resection; Cl, confidence interval; BMI, body mass index; AFP, $\alpha$-fetoprotein; HBsAg, hepatitis B surface antigen; HCV, hepatitis $C$ virus; MELD, model for end-stage liver disease; ALBI, albumin-bilirubin; PT INR, prothrombin time/international normalized ratio; HgB, hemoglobulin; ALT, alanine aminotransferase; ALKP, alkaline phosphatase.

could provide a survival benefit relative to TACE. Hence, SR is the recommended treatment in this clinical setting. Our study further validated that, even for patients with HCC tumor size exceeding $10 \mathrm{~cm}$, SR could proffer better survival than could TACE. A further liberal step beyond current guideline-charted territory of SR may be justified.

Conventional assessment of liver reserve, the ChildPugh classification, is not sufficiently accurate because the extents of ascites and encephalopathy were subjective variables (34). Furthermore, Child-Pugh class was originally designed for cirrhotic patients, and its use in patients with HCC prognosis may be inaccurate (35). Accordingly, Johnson recently proposed that ALBI scores, which incorporated both serum albumin and bilirubin levels, could provide a simple, objective, and evidencebased method for the evaluation of liver function for patients with liver cirrhosis or HCC (36). Moreover, it has been validated to accurately predict the prognoses of patients with HCC across different tumor stages and treatment modalities (34-41).

In our cohort, although most patients fell in the ChildPugh class A (96.1\%), only $37.3 \%$ of the patients were 
classified as ALBI grade 1. For these patients, ALBI grade still presented as an excellent predictor of prognosis. For patients with ALBI grade 1, the 5-year OS rate after SR was as high as $58.0 \%$. This suggests that, for patients with solitary huge HCC and ALBI grade 1 liver reserve, SR could be encouraged as the front-line treatment rather than be restricted to guideline-endorsed treatment, given that resection is highly likely to yield favorable long-term outcomes.

By the current concept, SR is a curative treatment modality while TACE is regarded as a non-curative local regional therapy for the treatment of HCC. Nevertheless, for patients with solitary huge HCC, substantial patients received TACE because of the concerns of safety and treatment efficacy of SR due to large tumor burden and the presence of microvascular invasion. In our cohort, no patient died within 30 days after the operation in the SR group. Moreover, the 90-day morality rates were also lower in the SR group when compared to that in the TACE group. Besides, the OS rate was also better in the SR group even given in the fact that most of the patients who underwent SR had microscopic invasion. In suggested that SR could be served as the front-line therapy for solitary huge HCC if the patients with a well-preserved liver function and R0 resection could be achieved.

There were some limitations to our study. First, the study was retrospective and, second, our institution is a tertiary referral medical center. Hence, selection bias cannot be ruled out. Third, SR is—at minimum-a partially operatordependent intervention. Our institutional experience may deviate from that of other institutions. Fourth, approximately $60 \%$ of patients had chronic HBV infection. In Taiwan, HBV is the major viral factor attributed to HCC carcinogenesis. On the other hand, chronic HCV infection accounts for the majority of HCC in Western countries, as patients with $\mathrm{HBV}$-induced HCC might have a lower rate of cirrhosis and better liver functional reserve relative to HCV-related HCC $(42,43)$. Conversely, regarding tumor factors, patients with HBV-related HCC seemed to have a higher rate of harboring gene signatures in the proliferation class, which was associated with poor prognosis compared to HCV-related HCC (1). Consequently, whether different initial carcinogenetic factors resulted in identical disease course and prognosis remains to be elucidated. Hence, generalizations of our results should remain conservative, with external validation from other cohorts highly encouraged. Fifth, several new treatment modalities, such as drug-eluting beads TACE, selective internal radiation therapy, molecular target therapy, and immune checkpoint inhibitors have been applied for patients with large HCC (1). Further prospective studies are warranted to assess the treatment efficacy and prognosis of these new treatment modalities for patients with solitary huge HCC.

In conclusion, for patients with solitary huge HCC, absence of major portal vein invasion, and no extra-hepatic metastasis, SR resulted in better survival than did TACE.

\section{Acknowledgments}

Part of this study was presented as a poster presentation at the 2018 annual meeting of the European Association for the Study of the Liver, Paris, France, April 11-15, 2018. Writing Assistance: American Manuscript Editors.

Funding: This work was supported by grants from the Ministry of Science and Technology of Taiwan (MOST 1062314-B-038-030, MOST 108-2314-B-075-049-MY3), and Taipei Veterans General Hospital (V107C-113, V108C-050, V107D36-003-MY2-2, VGHUST108-G6-1-2, Center of Excellence for Cancer Research MOHW107TDU-B-211-114019, and Big Data Center).

\section{Footnote}

Conflicts of Interest: Chien-Wei Su: Speakers' bureau: Gilead Sciences, Bristol-Myers Squibb, AbbVie, Bayer, and Roche. Advisory arrangements: Gilead Sciences. Other authors have no conflicts of interest to declare.

Ethical Statement: The authors are accountable for all aspects of the work in ensuring that questions related to the accuracy or integrity of any part of the work are appropriately investigated and resolved. The study was conducted in accordance with the Declaration of Helsinki and current ethical guidelines. It was approved by the Institutional Review Board (IRB) of the Taipei Veterans General Hospital. Informed consent was obtained before the patient underwent SR or TACE.

Open Access Statement: This is an Open Access article distributed in accordance with the Creative Commons Attribution-NonCommercial-NoDerivs 4.0 International License (CC BY-NC-ND 4.0), which permits the noncommercial replication and distribution of the article with the strict proviso that no changes or edits are made and the original work is properly cited (including links to both the formal publication through the relevant DOI and the license). 
See: https://creativecommons.org/licenses/by-nc-nd/4.0/.

\section{References}

1. Villanueva A. Hepatocellular Carcinoma. N Engl J Med 2019;380:1450-62.

2. Torre LA, Bray F, Siegel RL, et al. Global cancer statistics, 2012. CA Cancer J Clin 2015;65:87-108.

3. European Association For The Study Of The Liver, European Organisation For Research And Treatment Of Cancer. EASL-EORTC clinical practice guidelines: management of hepatocellular carcinoma. J Hepatol 2012;56:908-43.

4. Mazzaferro V, Regalia E, Doci R, et al. Liver transplantation for the treatment of small hepatocellular carcinomas in patients with cirrhosis. N Engl J Med 1996;334:693-9.

5. Kamo N, Kaido T, Yagi S, et al. Liver Transplantation for Intermediate-Stage Hepatocellular Carcinoma. Liver Cancer 2018;7:179-89.

6. Forner A, Reig M, Bruix J. Hepatocellular carcinoma. Lancet 2018;391:1301-14.

7. Kluger MD, Salceda JA, Laurent A, et al. Liver resection for hepatocellular carcinoma in 313 Western patients: Tumor biology and underlying liver rather than tumor size drive prognosis. J Hepatol 2015;62:1131-40.

8. Su CW, Chau GY, Hung HH, et al. Impact of steatosis on prognosis of patients with early-stage hepatocellular carcinoma after hepatic resection. Ann Surg Oncol 2015;22:2253-61.

9. Chen PC, Fang KC, Kao WY, et al. Outcomes of resection surgery for hepatocellular carcinoma: Does size matter? J Chin Med Assoc 2018;81:89-91.

10. Chan AWH, Zhong J, Berhane S, et al. Development of pre and post-operative models to predict early recurrence of hepatocellular carcinoma after surgical resection. J Hepatol 2018;69:1284-93.

11. Hwang S, Lee YJ, Kim KH, et al. The impact of tumor size on long-term survival outcomes after resection of solitary hepatocellular carcinoma: Single-institution experience with 2558 patients. J Gastrointest Surg 2015;19:1281-90.

12. Surveillance group; Diagnosis group; Staging group; Surgery group; Local ablation group; TACE/TARE/ HAI group; Target therapy/systemic therapy group; Radiotherapy group; Prevention group; Drafting group. Management consensus guideline for hepatocellular carcinoma: 2016 updated by the Taiwan Liver Cancer
Association and the Gastroenterological Society of Taiwan. J Formos Med Assoc 2018;117:381-403.

13. Zhou J, Sun HC, Wang Z, et al. Guidelines for diagnosis and treatment of primary liver cancer in China (2017 Edition). Liver Cancer 2018;7:235-60.

14. European Association for the Study of the Liver. EASL Clinical Practice Guidelines: Management of hepatocellular carcinoma. J Hepatol 2018;69:182-236.

15. Kokudo T, Hasegawa K, Yamamoto S, et al. Surgical treatment of hepatocellular carcinoma associated with hepatic vein tumor thrombosis. J Hepatol 2014;61:583-8.

16. Ishizawa T, Hasegawa K, Aoki T, et al. Neither multiple tumors nor portal hypertension are surgical contraindications for hepatocellular carcinoma. Gastroenterology 2008;134:1908-16.

17. Chang WT, Kao WY, Chau GY, et al. Hepatic resection can provide long-term survival of patients with non-earlystage hepatocellular carcinoma: extending the indication for resection? Surgery 2012;152:809-20.

18. Torzilli G, Belghiti J, Kokudo N, et al. A snapshot of the effective indications and results of surgery for hepatocellular carcinoma in tertiary referral centers: is it adherent to the EASL/AASLD recommendations?: an observational study of the HCC East-West study group. Ann Surg 2013;257:929-37.

19. Roayaie S, Jibara G, Tabrizian P, et al. The role of hepatic resection in the treatment of hepatocellular cancer. Hepatology 2015;62:440-51.

20. Zhong JH, Ke Y, Gong WF, et al. Hepatic resection associated with good survival for selected patients with intermediate and advanced-stage hepatocellular carcinoma. Ann Surg 2014;260:329-40.

21. Vitale A, Burra P, Frigo AC, et al. Survival benefit of liver resection for patients with hepatocellular carcinoma across different Barcelona Clinic Liver Cancer stages: a multicentre study. J Hepatol 2015;62:617-24.

22. Cucchetti A, Cescon M, Golfieri R, et al. Hepatic venous pressure gradient in the preoperative assessment of patients with resectable hepatocellular carcinoma. J Hepatol 2016;64:79-86.

23. Yau T, Tang VY, Yao TJ, et al. Development of Hong Kong Liver Cancer staging system with treatment stratification for patients with hepatocellular carcinoma. Gastroenterology 2014;146:1691-700.e3.

24. Bruix J, Sherman M. Management of hepatocellular carcinoma: an update. Hepatology 2011;53:1020-2.

25. Hsieh WY, Chen PH, Lin IY, et al. The impact of esophagogastric varices on the prognosis of patients with 
hepatocellular carcinoma. Sci Rep 2017;7:42577.

26. Fang KC, Kao WY, Su CW, et al. The prognosis of single large hepatocellular carcinoma was distinct from Barcelona Clinic Liver Cancer Stage A or B: The role of albuminbilirubin grade. Liver Cancer 2018;7:335-58.

27. D'Agostino RB, Jr. Propensity score methods for bias reduction in the comparison of a treatment to a nonrandomized control group. Stat Med 1998;17:2265-81.

28. Hung HH, Chiou YY, Hsia CY, et al. Survival rates are comparable after radiofrequency ablation or surgery in patients with small hepatocellular carcinomas. Clin Gastroenterol Hepatol 2011;9:79-86.

29. Chang CY, Hsieh WY, Chau GY, et al. Esophageal varices are not predictive of patient prognosis after surgical resection of hepatocellular carcinoma. Eur J Gastroenterol Hepatol 2018;30:1368-77.

30. Hsu CY, Liu PH, Hsia CY, et al. Surgical resection is better than transarterial chemoembolization for patients with hepatocellular carcinoma beyond the Milan criteria: A prognostic nomogram study. Ann Surg Oncol 2016;23:994-1002.

31. Yamashita Y, Taketomi A, Shirabe K, et al. Outcomes of hepatic resection for huge hepatocellular carcinoma $(>/=$ $10 \mathrm{~cm}$ in diameter). J Surg Oncol 2011;104:292-8.

32. Zhu SL, Zhong JH, Ke Y, et al. Efficacy of hepatic resection vs transarterial chemoembolization for solitary huge hepatocellular carcinoma. World J Gastroenterol 2015;21:9630-7.

33. Shrager B, Jibara GA, Tabrizian P, et al. Resection of large hepatocellular carcinoma $(>/=10 \mathrm{~cm})$ : a unique western perspective. J Surg Oncol 2013;107:111-7.

34. Kao WY, Su CW, Chiou YY, et al. Hepatocellular carcinoma: nomograms based on the albumin-bilirubin grade to assess the outcomes of radiofrequency ablation. Radiology 2017;285:670-80.

35. Hiraoka A, Kumada T, Tsuji K, et al. Validation of

Cite this article as: Wei CY, Chen PC, Chau GY, Lee RC, Chen PH, Huo TI, Huang YH, Su YH, Hou MC, Wu JC, $\mathrm{Su}$ CW. Comparison of prognosis between surgical resection and transarterial chemoembolization for patients with solitary huge hepatocellular carcinoma. Ann Transl Med 2020;8(5):238. doi: $10.21037 /$ atm.2019.12.157 modified ALBI grade for more detailed assessment of hepatic function in hepatocellular carcinoma patients: A multicenter analysis. Liver Cancer 2019;8:121-9.

36. Johnson PJ, Berhane S, Kagebayashi C, et al. Assessment of liver function in patients with hepatocellular carcinoma: A new evidence-based approach-The ALBI grade. J Clin Oncol 2015;33:550-8.

37. Chen PC, Chiu NC, Su CW, et al. Albumin-bilirubin grade may determine the outcomes of patients with very early stage hepatocellular carcinoma after radiofrequency ablation therapy. J Chin Med Assoc 2019;82:2-10.

38. Chen PH, Hsieh WY, Su CW, et al. Combination of albumin-bilirubin grade and platelets to predict a compensated patient with hepatocellular carcinoma who does not require endoscopic screening for esophageal varices. Gastrointest Endosc 2018;88:230-239.e2.

39. Pinato DJ, Sharma R, Allara E, et al. The ALBI grade provides objective hepatic reserve estimation across each BCLC stage of hepatocellular carcinoma. J Hepatol 2017;66:338-46.

40. Chan AW, Kumada T, Toyoda H, et al. Integration of albumin-bilirubin (ALBI) score into Barcelona Clinic Liver Cancer (BCLC) system for hepatocellular carcinoma. J Gastroenterol Hepatol 2016;31:1300-6.

41. Lee PC, Chen Y'T, Chao Y, et al. Validation of the albumin-bilirubin grade-based integrated model as a predictor for sorafenib-failed hepatocellular carcinoma. Liver Int 2018;38:321-30.

42. Kao WY, Su CW, Chau GY, et al. A comparison of prognosis between patients with hepatitis B and C virusrelated hepatocellular carcinoma undergoing resection surgery. World J Surg 2011;35:858-67.

43. Kulik L, El-Serag HB. Epidemiology and management of hepatocellular carcinoma. Gastroenterology 2019;156:477-491.e1. 